\title{
Anomalous bond stretching phonons as a probe of charge fluctuations in perovskites
}

\author{
S. Cojocaru ${ }^{\text {a,c,* }}$ R. Citro ${ }^{\text {a }}$ M. Marinaro ${ }^{\text {a,b }}$ \\ a Dipartimento di Fisica "E. R. Caianiello", Universitá degli Studi di Salerno and CNISM, Unitá di ricerca di Salerno, Via \\ S. Allende, 84081 Baronissi (SA), Italy \\ b I.I.A.S.S., Via G. Pellegrino, n. 1984019 Vietri sul Mare (SA), Italy \\ ${ }^{\mathrm{c}}$ Institute of Applied Physics, Chisinau 2028, Moldova
}

\begin{abstract}
Important information on momentum resolved low energy charge response can be extracted from anomalous properties of bond stretching in plane phonons observed in inelastic neutron and X-ray scattering in cuprates and some other perovskites. We discuss a semiphenomenological model based on coupling of phonons to a single charge mode. The phonon dispersion and linewidth allow to locate the energy of the charge excitation in the mid infrared part of the spectrum and to determine some of its characteristics. New experiments on oxygen isotope substitution could allow to achieve a more detailed description. Corresponding relations following from the model can be used for the interpretation of experiments and as test of the model.
\end{abstract}

\section{Introduction}

A direct coupling of the bond stretching (BS) inplane $\left(\mathrm{CuO}_{2}\right)$ phonon modes to density fluctuations of strongly correlated electrons has been discussed as a possible reason of their anomalous properties [1],[2]. Doping dependent anomalies of these high energy $(70-90 \mathrm{meV})$ phonon branches are observed in a number of perovskite materials like cuprates, bismuthates, manganites, nickelates [3]-[7]. In particular, presence of a strong (more than 20\%) softening of the BS phonon dispersion towards the Brillouin zone boundary in the (100) and (010) directions contrasts a very week dispersion in the diagonal direction (110). This is paralleled by the phonon linewidth that reaches unusually large values too, $10 \mathrm{meV}$, in the (100) and (010). In some materials softening can also be non-monotonous and one

\footnotetext{
* Corresponding author.

Email addresses: cojocaru@sa.infn.it (S. Cojocaru ), citro@sa.infn.it (R. Citro), marinaro@sa.infn.it (M. Marinaro).
}

observes a minimum at intermediate momenta, e.g. $q \sim 0.3$ r.l.u. A number of theories and models have been developed to explain the properties of BS phonons. To mention a few possible scenarios, such as incommensurate charge density wave instability or stripes, acoustic and optical plasmons or unusual dielectric properties (overscreening, when the dielectric constant becomes negative at some momenta) as well as calculations based on ab-initio LDA, e.g. [8], or strong electron correlations, e.g. [9]. Different scenarios refer to different energy scale of the charge excitations coupled to BS phonons. In most cases its direct experimental measurement is not available, because it requires a sufficient momentum resolution at relatively low energies. Most of the present knowledge comes from optical experiments, i.e. small momentum $q$, where the coupling to the BS phonon mode is vanishing. In this context it seems interesting to consider the phonon itself as a probe of the charge excitation. We employ a semiphenomenological model where the charge response coupled to the phonon is parametrized in a 
simple form. To determine all three parameters some additional measurements of the BS mode properties are required. We propose to consider dependence on oxygen isotope substitution of phonon dispersion and linewidth. This would allow not only to determine the parameters of the model but also to test its validity, since the number of equations is larger than required by selfconsistency.

\section{Model and results}

The Dyson equation for the renormalized phonon propagator can be written in the form

$\omega_{0} D^{-1}(q, \omega)=\omega^{2}-\omega_{0}^{2}\left(1+\alpha \sin ^{2}\left(q_{x} / 2\right) P(q, \omega)\right)$

where the structure factor corresponds to (100), $\omega_{0}$ is the bare frequency, $\alpha$ is the coupling constant [1] and $P(q, \omega)$ the charge susceptibility. The latter is taken in the Lorentzian form

$P(q, \omega)=\frac{\eta_{q}}{\omega^{2}-\Omega_{q}^{2}+i \Gamma_{q} \omega}$,

with unknown dispersion $\Omega_{q}$, linewidth $\Gamma_{q}$ and oscillator strength $\eta_{q}$. The model (1), (2) defines the equation for the phonon frequency

$\omega_{q}^{2}=\omega_{0}^{2}\left(1+\beta_{q} \frac{\sin ^{2}\left(q_{x} / 2\right)\left(\omega_{q}^{2}-\Omega_{q}^{2}\right)}{\left(\omega_{q}^{2}-\Omega_{q}^{2}\right)^{2}+\left(\Gamma_{q} \omega_{q}\right)^{2}}\right)$,

and linewidth

$\gamma_{q}=\Gamma_{q} \frac{\omega_{0}^{2}-\omega_{q}^{2}}{\Omega_{q}^{2}-\omega_{q}^{2}}$

where $\beta_{q}=\alpha \eta_{q}$ is the effective coupling constant. Analysis of above equations shows that according to observed features of the BS softening, dispersion $\Omega_{q}$ along (100) and (010) should be much smaller than in diagonal direction, that agrees with results based on $t-J$ model [1]. We then estimate the values of $\Gamma_{q}$ by substituting known data on $\omega_{q}$ and $\gamma_{q}$ into (4) and changing the values of $\Omega_{q}$. After these results are confronted with data on optical absorption, it follows that the relevant charge excitation should be located in the mid infrared region, $0.2-0.6 \mathrm{eV}$. This is further corroborated by the analysis of its doping dependence in some cuprates [10] as compared to softening of the BS phonons.

Additional information can be extracted by considering the effect of the isotope mass shift $d m=$ $m\left(O^{18}\right)-m\left(O^{16}\right)$ on dispersion $d \omega_{q}=\omega_{q}\left(O^{18}\right)-$
$\omega_{q}\left(O^{16}\right)$ and linewidth $d \gamma_{q}$. In particular, the model yields

$d \omega_{q} / d \omega_{0} \simeq \sqrt{1-\beta_{q} \sin ^{2}\left(q_{x} / 2\right) / \Omega_{q}^{2}}$,

$d \gamma_{q} / d \omega_{0} \simeq 2 \gamma_{q} / \omega_{0}$.

in the lowest order of the $\omega_{0} / \Omega_{q}$ expansion. We further define the momentum dependent isotope coefficient $\alpha_{q} \equiv-\left(m d \omega_{q} / \omega_{q} d m\right)$ that allows to introduce a new relation to the parameters of the charge excitation derived in the same approximation

$\alpha_{q}-\alpha_{0}=\alpha_{0} \beta_{q}\left(\Gamma_{q}^{2} / \Omega_{q}^{2}-1\right) \sin ^{2}\left(q_{x} / 2\right) \omega_{0}^{2} / \Omega_{q}^{4}$

If $\omega_{0} / \Omega_{q}$ is not small, i.e. dynamic effects are impor(1) tant, the full expressions should be used (not given here).

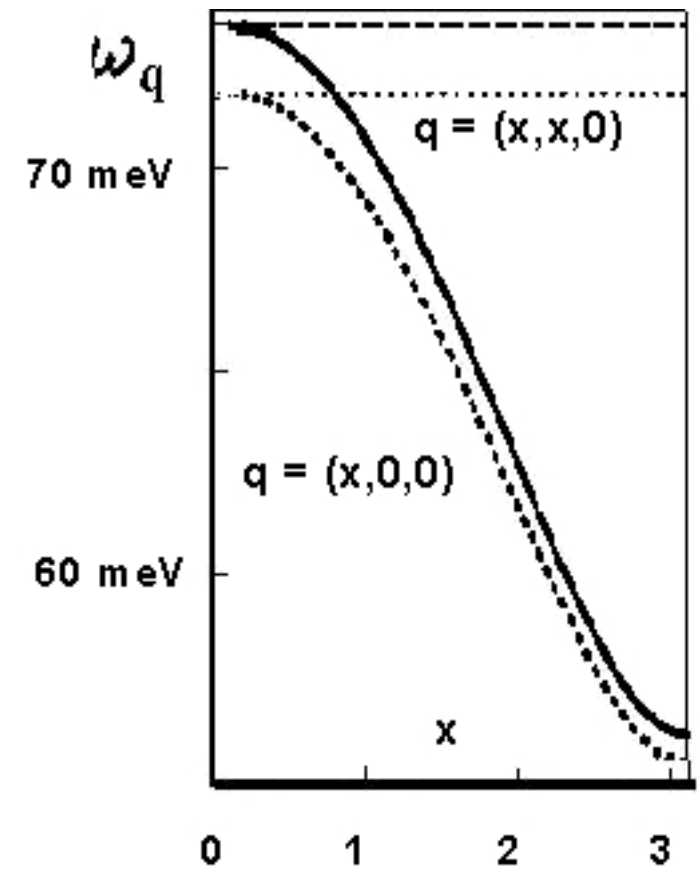

Fig. 1. The expected isotope dependence of phonon dispersion along the two directions in the Brillouin zone.

Eqs. (3-7) contain the relation between experimentally measurable characteristics of the phonon spectrum and the parameters of the model describing the charge excitation. On the basis of available data the model predicts that the energy of charge excitation responsible for the renormalization of the BS phonon mode is located in the mid infrared region. Its energy is lowered with hole doping and respectively its effect on the BS phonon increases. To be noted that the sign of the differential isotope coefficient (7) depends on the ratio of $\Omega_{q}$ 
to the linewidth. We expect that the fingerprint of the broad MIR structure will show up in a positive value of this coefficient. Another observation is that phonon linewidth could be a more sensitive probe of the isotope effect than phonon dispersion as seen from (5) and (6).

\section{References}

[1] P. Horsch and G. Khaliullin, Physica B, 359361, 620 (2005); D.N. Aristov and G. Khaliullin cond-mat/0603161 (and references therein).

[2] O. Rosch and O. Gunnarsson, Phys. Rev. Lett. 93, 237001 (2004).

[3] L. Pintschovius, Phys. Stat. Sol. B 242, 30 (2005).

[4] T. Fukuda et al., Phys. Rev. B 71, 060501 (2005).

[5] M. Braden et al., Physica C, 378-381, 89 (2002).

[6] J.M. Tranquada et al., Phys. Rev. Lett. 88, 075505 (2002).

[7] D. Reznik et al., Nature 440, 1170 (2006).

[8] C. Falter, Phys. Stat. Sol. B 242, 78 (2005).

[9] M. L. Kulić and O. V. Dolgov, Phys. Rev. 71, 092505 (2005).

[10] F. Hanke and M. Azzouz, M. J. Cond. Matt., 6, 1 (2005); http://www.fsr.ac.ma/MJCM/vol6-art01.pdf 